\title{
ELEMENTS ITALIENS DU LEXIQUE ISTROROUMAIN
}

La péninsule d'Istrie est une aire de contacts linguistiques millénaires entre les idiomes romans et les idiomes slaves. Dès leur arivée en Istrie au VII ${ }^{\mathrm{e}}$ s., les Croates et les Slovènes sont entrés en contact avec les populations romanes autochtones (les ancêtres des Istroromans dans le sud, les ancêtres des Frioulans dans l'arrière-pays de la ville de Trieste). C'est à partir du $\mathrm{X}^{\mathrm{e}}$ s. que commence, en Istrie, une expansion politique et linguistique vénitienne et un contact permanent entre les langues slaves et différentes formes de la langue italienne, en premier lieu avec le dialecte vénitien. Parmi les populations qui, à la fin du $\mathrm{XV}^{\mathrm{e}}$ s., affluaient en Istrie du nord de Dalmatie, il y avait, entre autres, un certain nombre de sujets parlant une variété de la langue rounaine qui, plus tard, sera désignée par les romanistes comme istroroumain.

C'est sur la base de ces faits que le professeur Pavao Tekavčić proposait - dans un article publié en 1976 (SRAZ, $\mathrm{N}^{\mathrm{O}}$ 41-42, pp. 227-240) - l'élaboration d'un A tlas linguistique d'Istrie qui permettrait une étude systématique des emprunts et calques linguistiques et des stratifications lexicales dans différents idiomes d'Istrie (Tekavčić, 1976, 227). La nature et l'intensité des contacts entre les cinq idiomes en cause (les idiomes slaves: le croate et le slovène; les idiomes romans: l'italien, l'istroroman et l'istroroumain) dépendent des conditions d'ordre social, économique, politique, culturel etc. Langues d'enclave et langues d'un prestige réduit, l'istroroman et l'istroroumain ont exercé une influence presque insignifiante sur les autres idiomes. En tant que langue d'une grande puissance coloniale pendant presque un millénaire, en tant que langue de première importance internationale et qui disposait d'un grand nombre d'institutions, la langue italienne (au début, avant tout sous forme de dialecte vénitien) a laissé de nombreuses traces dans tous les idiomes d'Istrie. Le croate sur le territoire de la presqu'île proprement dite et le slovène dans sa périphérie du nord s'imposaient surtout par une masse importante de sujets parlants; ce n'est qu'à partir du XIX siècle que ces deux langues commencent à agir par l'intermédiare de toute une série d'institutions culturelles et administratives et en tant que véritables 'langues nationales'.

Les villages istroroumains représentent une sorte d'enclave à l'intérieur du territoire compact de langue croate. A part quelques exceptions parmi les enfants d'âge préscolaire, toute la population istroroumaine est bilingue; à côté de l'istroroumain, elle se sert activement aussi du croate (Petrovici et Neiescou, 1965, 352-3; Kovačec, 1968, 80). Ce n'est qu'au village de Žeiân (dans le nord) qu'on peut rencontrer des personnes possédant des connaissances du slovène. Un certain nombre de personnes adultes (avant tout les hommes, plus particulièrement dans les villages du sud) ma- 
nient aussi la langue italienne, qu'il s'agisse de la koiné vénitienne d'Istrie ou de l'italien standard. Par conséquent, le croate est une composante obligatoire du bilinguisme (/plurilinguisme) des Istroroumains (Kovačec, 1984, 551).

Les contacts entre l'istroroumain et l'italien sont très anciens, mais dans la région de Ćićarija (dans le village isolé de Žeiân) ils ont depuis toujours été sporadiques et superficiels. Les villages istroroumains du sud (Sušńéviţę, Nóselo, Bârdo, Letåi etc.) faisaient partie du comté de Pazin qui, pratiquement de tous les côtés, était entouré des domaines de 1'Istrie Vénitienne' où la langue italienne bénéficiait d'un statut de langue officielle, langue des relations publiques, langue de culture (Kovačec, 1984, 552) et où une partie de la population au moins ne parlait d'autre langue que l'italien (surtout sous sa forme vénitienne). C'est grâce aux contacts assez fréquents et relativement réguliers avec la population italophone que les Istroroumains du sud ont adopté, dans leur langue, un bon nombre de mots italiens. Au cours du XIX ${ }^{e}$ s., et surtout pendant l'occupation italienne de l'Istrie (1918-1943), l'importance de l'influence linguistique italienne sur l'istroroumain n'a fait qu' augmenter.

L'istroroumain et le croate d'Istrie étaient exposés au même type d'influence italienne pendant plusieurs siècles et dans des conditions plus ou moins identiques. La conséquence en est que l'istroroumain et le croate ont emprunté à l'italien presque les mêmes unités lexicales. Cependant, si l'on tient compte du fait que tous les Istroroumains parlent croate et qu'il n'y a qu'un petit nombre de Croates capables de se servir de l'istroroumain, si l'on prend en considération aussi le fait qu'une partie seulement des Croates et des Istroroumains arrivent à manier l'italien d'une manière active, on pourra conclure qu'une partie des emprunts à l'italien sont entrés en istroroumain par l'intermédiaire de la langue croate (Puşcariu, 1926, 223; Kovačec, 1971, $197 ; 1984,552$; Flora, 1975, 56). On peut supposer qu'un emprunt à la langue italienne se maintiendra en istroroumain plus facilement s'il existe en croate. La preuve en est aussi le fait que - en ce qui concerne les emprunts à l'italien - entre le croate de Ćićarija et le croate au sud de la montagne Učka on pourrait établir le même type de rapports qu'entre l'istroroumain du nord et l'istroroumain du sud.

Il faut souligner qu'entre les Istroroumains du sud et les Istroroumains du village de Žeiân il n'y a pratiquement pas de contacts. Le résultat de cette évolution indépendante et divergente des deux variétés de l'istroroumain est un certain nombre de différences, entre les deux types linguistiques, dans tous les compartiments de la langue. Ces différences sont particulièrement importantes justement par rapport au nombre et au rôle des éléments d'origine italienne: dans les parlers du sud, le nombre des emprunts à l'italien dépasse plusieurs fois leur nombre dans le parler de Žeiân.

Dans les lignes qui suivent, nous allons présenter les emprunts istroroumains à l'italien, classés par groupes sémantiques (pour les substantifs) ou suivant les parties du discours auxquelles ils appartiennent.

Les emprunts à l'italien désignant des phénomènes atmosphériques sont peu nombreux en istroroumain. Le mot årije 'air' est caractéristique pour les parlers du 
sud; dans le parler de Žeiân on emploie, avec la même signification, le mot d'origine croate $z$ råc, le terme proprement roumain åier étant réservé pour la signification 'tourbillon del l'air'. On peut mentionner aussi deux noms de vents: bórę 'bora' (Žeiân: bóra) et tramuntåne 'tramontane'.

Termes designant des unités de temps. Le mot úrę (Ž.: úra) 'heure' est emprunté par l'intermédiaire du croate (cf. Skok, 1972, 562). Les termes setimåne 'semaine' (à Žeiân presque exclusivement le mot d'origine croate $t^{\prime \prime} e ́ d a ̂ n$ ), primavérę (à côté de prolitt"e, mládo léto, <cr.) et autúno 'automne' (à côté de jésen, pozímâc, < cr.) sont typiques pour les villages du sud; le mot setimana est courant dans les parlers croates de la région tandis que les deux autres n'y apparaissent pas. A côté des noms de mois empruntés à l'italien, par la langue croate, à une époque plus ancienne (ienår 'janvier', Skok, 1971, 754; febrår 'février', Skok, 1971, 508; setémbâr 'septembre' etc.), on trouve dans les parlers du sud aussi quelques emprunts directs comme apríle 'avril' et otóbre 'octobre'.

Noms d'animaux. En istroroumain, les noms d'animaux empruntés à l'italien sont peu nombreux. Il s'agit de deux noms d'oiseaux - rondón '(une espèce d') hirondelle, Hirundo Apus' (it. rondone, Zingarelli, p. 1658; vén. rondòn, Boerio, p. 583) et catóme 'Tetrao rufus' (cf. vén. cotórno, Boerio, 205) et du substantif šcróva (Ž. désignant la 'femelle du porc, truie' (ce mot s'explique par un emprunt au vénitien scrova, Boerio, 636, ou éventuellement au frioulan scròve, Pirona, 990). Le nom d'un poisson, bacalå 'morue séchée, stockfish', répandu aussi dans les parlers croates d'Istrie, appartient en réalité aux emprunts désignant des aliments.

Noms de plantes. Les noms des plantes sauvages empruntés à l'italien sont peu nombreux en istroroumain. Nous signalons les noms pin 'pin', brúla '(une espèce de) jonc' (cf. brùla, Boerio, 102) et palúd '(l'ensemble des) végétaux d' un marécage'. Il semble que les mots brúla et palúd ont été apportés par les ouvriers italiens qui, entre les deux guerres, ont pris part à l'assainement du lac (/marécage) de Čepić.

En Istrie, la plupart des innovations dans le domaine de l'agriculture se propageaient du nord d'Italie. C'est pourquoi, en istroroumain et dans les parlers croates d'Istrie, on trouve un grand nombre de noms de plantes cultivées qui sont empruntés à l'italien. Le mot pour 'maïs' est, dans les parlers istroroumains du sud, une traduction croate approximative de l'expression italienne grano turco, granturco ('grano esotico, forestiero', cf. Zingarelli, 843, 2070), trukíne (en croate trukinja '(la) turque'), et dans le parler de Žeiân un emprunt à l'italien fâmentín (avec changement de suffixe; cf. formentòn, Boerio, 282; frumentone, formentone, Zingarelli, 753, 772). Deux plantes fourragères répandues en Istrie sont désignées par des noms d'origine italienne: jårrba špåńa 'luzerne' (à côté du nom croate dítelinę; mais le croate d'Istrie se sert aussi du nom emprunté à l'italien erbašpanja) et trefoíón 'trèfle'. Pour 'plante ornementale' l'istroroumain du sud emploie tout simplement le mot italien piånte (it. pianta). Les '(petits) pois' (Pisum sativum) sont désignés par le mot bízi (sud), bíži (Žeiân), emprunté au vénitien biso, bisi (Boerio, 83; it. pisello), et le 'haricot' par le mot fazó (sud), fažó (Ž.) (forme déterminée: fazólu, fažólu, cf. vén. 
fasòlo, Boerio, 262; it. fagiolo). Comme terme d'ensemble pour les 'légumes', l'istroroumain emploie le mot emprunté à l'italien verdúrę et, de la même façon, pour exprimer la notion générique des 'fruits' il se sert du mot d'origine italienne frúti (pl.;

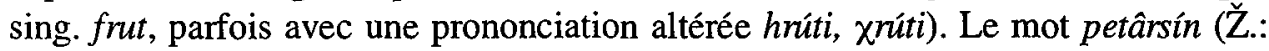
petâršín) 'persil' remonte, par de nombreuses formes intermédiaires croates, au vénitien petersemolo (Skok, 1972, 643), le mot mérlin 'carrote' a été emprunté au triest. mérlino (d'origine allemande; Skok, 1972, 409), carótę 'radis noir' à l'italien (caròta), pése 'betterave (rouge)' (mot courant en croate d'Istrie et en slovène) remonte au lat. beta à travers le vieux-haut-allemand pie $3 a$ (Skok, 1971, 164). Le mot capúz 'chou' est un élément vénitien très répandu en croate (cf. capuzzo, Boerio, 135), le terme pomidór (pl. pomidóri) 'tomate' remonte à une forme italienne populaire pomidòro (pl. pomidòri, Zingarelli, 1431; vén. pomi d'oro, Boerio, 519). Les termes túke 'courge' et thukéte 'courgette' ont probablement été empruntés au vénitien (cf. Boerio, zuca, 822, zuchèta, 823). Les mots pour désigner les 'concombres' cucú$\operatorname{mar}(i)(\check{Z}$.) remontent, par l'intermédiaire du croate, au vénitien cucùmaro (it. cocòmero). Enfin, sont d'origine italienne les noms de deux plantes à fruits très appréciés, melón 'melon' et angúrije 'pastèque.

Parmi les arbres fruitiers, le nom du 'marronnier', manín (comme en croate, le nom désigne aussi le fruit), est emprunté à l'italien. Un type spécial de cultiver la vigne 'treille, pergola', qui, en Istrie, s'est propagé de l'Italie du nord, porte un nom emprunté à l'italien du nord bråădi (pl.; sg. brånde). Enfin, la 'récolte', les 'produits agricoles ou les fruits d'une année', que les Istroroumains et les Croates vendaient très souvent à la population de langue italienne des villes d'Istrie, est désignée par un emprunt au vénitien intråda (cf. intràda, Boerio, 350; Skok, 1971, 727).

Noms des aliments, plats et repas, boissons etc. Pays avec une tradition culinaire millénaire et avec une culture de la nourriture et des boissons ancienne, Italie fornissait des produits de son art alimentaire et culinaire toute l'Istrie, et un nombre important de termes italiens relatifs à ce domaine a pénétré en croate et en istroroumain. C'est à cause du plus grand prestige de l'italien, ou parfois tout simplement à cause de la mode, que les termes italiens ont éliminé les termes croates, istroroumains ou slovènes plus anciens. A côté du terme croate måslo 'beurre', l'istroroumain du sud a emprunté aussi les mots italiens butíro et búro (à Žeiân: unt, roum.). Les produits d'épicerie, qui ont pénétré en Istrie par l'intermédiaire des villes de l'Italie du nord, portent en istroroumain (comme en croate et en slovène) les noms empruntés par l'intermédiaire de la langue italienne, p. ex. café 'café' (forme déterminée: cafélu, sud, cafétu, Ž.; cf. aussi: colór café) et oríz 'riz'. La 'fleur de farine' est désignée en istroroumain par un mot emprunté au vénitien fíorét (vén. fioréto, Boerio, 274). D'une manière semblable, le 'petit pain', en tant que produit 'de luxe' qui provenait des villes, porte un nom emprunté à l'italien litt. panín (it. panino). Les 'pâtes' sont un des produits alimentaires d'Italie les plus connus et l'istroroumain (aussi bien que le croate d'Istrie) désigne les 'pâtes' par un mot emprunté à l'italien påste (sud; påšta, Ž.). Le mot it. polénta est répandu en istroroumain (paléntę, sud, palénta, Ž.) comme 
dans un grand nombre de parlers croates occidentaux (cf. Skok, 1972, 592). Le 'levain' est désigné en istroroumain par la forme críme (Ž.: críma) qui, à ce qu'il semble, aurait pu être empruntée à un parler de l'Italie du nord. Sont d'origine italienne aussi les mots súgo 'sauce', martadéle 'mortadelle', pârsút, pâršút 'jambon (cru)' (it. prosciutto), túcor 'sucre' (vén. zùcaro, avec changement de suffixe en croate). La 'soupe aux légumes et au riz' est désignée en istroroumain (comme en croate d'Istrie) par le mot manéstre, manéštra (Ž.) emprunté au vénitien (cf. manestra, Boerio, 393; it. minestra). Le mot pour 'casse-croûte, collation', maréndę, marénda, emprunté au vénitien (cf. marenda, Boerio, 393; it. merenda), est un doublet étymologique du mot roumain merínde qui se conserve dans le parler de Žeinân avec la signification 'déjeuner, repas du midi'. Parmi les noms de boissons, sont d'origine italienne bíre, bíra 'bière' et malvasíîe 'malvoisie, espèce de vin doux et aromatique'.

C'est par l'intermédiaire des villes de l'Italie du nord que s'est propagée en Istrie l'habitude de jouïr du tabac. En istroroumain (de la même manière qu'en croate $\mathrm{d}$ 'Istrie) sont d'origine italienne les termes comme tabåc 'tabac', špańuléti 'cigarettes' (cf. Skok, 1973, 409) et préze 'tabac à priser'.

Il n'y a en istroroumain que quelques termes désignant des parties du corps (ou formes des parties du corps) qui sont empruntés à l'italien. Un terme fréquent de ce type est stúmig (sud), štúmì (Ž.) 'poitrine' mais aussi 'estomac' (comme stòmego en vénitien; cf. Boerio, 706). Est généralement employé aussi le terme góbe

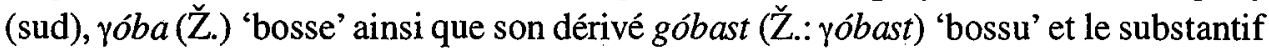
correspondant góbo, yóbo avec la signification '(le) bossu'. Sont très peu nombreux aussi les termes d'origine italienne qui désignent des maladies ou des états du corps. Parmi les mots les plus fréquents appartenant à cette catégorie on peut citer p. ex. oret"óni 'oreillons', malárịie 'paludisme, malaria' (un mot qui pourrait être, aussi, un emprunt au croate malàrija), brofúl' 'bulle, vésicule' et fébre 'fièvre'.

Parmi les emprunts istroroumains à l'italien on trouve quelques termes désignant des matières et matériaux etc. (ainsi que différentes formes des matières). Nous citons dans cette catégorie les mots comme cârbún 'charbon', salbún 'sable' (cf. Skok, 1973, 181), batúde 'pierres concassées, cailloutis', tavalón 'madrier', råm 'cuivre; airain', rúzine 'rouille' (vén. rùzene, Boerio, 586), petról'a, pitról'a, petróvel' (sud), petróliio (Ž.) 'petrole (à lampe)' etc. Il n'y a que quelques mots désignant des mesures, comme cvartín 'quart', tonolåte 'tonne', duzíne 'douzaine' (vén. dozèna, Boerio, 246); est apparenté à cette catégorie aussi le mot fašine, fašína 'fagot' . Dans le mot navil'åde (sud) 'fourchée', ce n'est que le suffixe qui est d'origine vénitienne (-ada), tandis que la partie lexicale du mot est un élément croate (na vile 'sur la fourche, avec la fourche'; à Žeiân, on trouve la forme navil'åi avec un suffixe croate $-a j$ ).

Pour Istrie, l'Italie du nord a depuis toujours été l'un des centres les plus importants d'où se propageaient différentes innovations techniques. C'est pourquoi un certain nombre de mots désignant des dispositifs techniques ou différentes machines 
est d'origine italienne, aussi bien en istroroumain que dans les parlers croates d'Istrie. Appartiennent à cette catégorie les mots comme måkine 'machine; dispositif; voiture' (Ž.: måkina), vetúrę 'voiture', caróte 'voiture, fiacre', biţicléte 'bicyclette' (peut-être, le résultat d'un croisement entre l'it. bicicletta, avec [ť̌], et le croate bicikl, avec [ts]; mais il faut retenir qu'on trouve, dans les parlers croates, biciklèta et bičiklèta), coriịére 'autocar', trebilatríče 'batteuse', ferovíie 'chemin de fer', acvedóto 'aqueduc', calún 'canon', broštulino 'brûloir', etc. Appartiennent au même domaine sémantique les noms de différents outils, instruments et accessoires, comme p. ex. según 'scie' (it. segone), tamize 'tamis', timún 'timon (de voiture)', péze 'balance, bascule', månig '(le) manche', maniţe '(le) manche, poignée', cavalét 'chevalet', låma (de fl'er) 'jante', šéšula 'écope; petite pelle’ (vén. sèssola, Boerio, 649), puntél 'étai, étançon', måțe 'masse', årme 'armes' etc. Sont apparentés à cette catégorie les mots désignant différents produits techniques ou materiel technique, comme p. ex. balín, balóte 'grain de plomb; boule', båle 'balle; ballot', brénte 'comporte' (cf. Skok, 1971, 207), cåibe 'cage', cåreg 'cartouche', caséte 'caisse, boîte', córde 'corde', ferål 'lanterne' (vén. feral, Boerio, 265), fârminånti, fuminånti 'allumettes' (it. fulminanti; pour fiammiferi), hårte 'papier', låpež, låpiž 'crayon', líbâr 'livre', masté 'baquet, cuve', medizį́e 'médicament' (dérivé à partir du vén. medesìna), sédiie 'selle', šcåtulę 'boîte', špåg 'ficelle', pasaréle 'passerelle' etc.

Termes désignant des pièces de la vaisselle et du couvert. Les pièces de la vaisselle et du service de table représentent les objets dont l'usage en Istrie a depuis toujours été influencé par les usages et les produits de l'Italie du nord. Appartiennent à ce groupe les mots comme våž 'vase', bote 'carafe' (cf. vén. bozza, Boerio, 96), kíkere 'tasse' (it. chicchera), piiåa 'assiette', scodéle 'bol; écuelle', padéle 'poêle', pináte 'marmite, pot', fugérę 'brasero' (vén. foghera, Boerio, 277). Pour dire 'couvert', l'istroroumain emploie le mot italien de forme vénitienne pošåd $\ell$ ou pašåd $\ell$ (cf. possada, Boerio, 528; it. posata), mais qui signifie en même temps 'couteau (du service de table)'; 'fourchette' se dit pinín.

Mots désignant les parties de la maison et les meubles. Avec la signification 'chambre', on emploie la forme cåmare au sud (vén. càmara, Skok, 1972, 24) et la forme cåmbra à Žeinân (ibid.); on peut citer encore les mots comme scåle, šcåle 'escalier'; štúfe 'poêle', fogolér 'foyer' (vén. fogoler, Boerio, 277), cantún 'coin' (Skok, 1972, 31-32), cundót 'lieux d'aisance' (Skok, 1972, 138) etc. Dans le parler de Žeiân on trouve le mot miza 'table', comme en slovène et dans certains parlers croates d'Istrie; il s'agit probablement d' un emprunt à l'italien du nord (Skok, 1972, 435). Le mot casetín 'tiroir' est emprunté à l'italien standard, aussi bien que le mot casún. L'élément treipičór 'trépied' représente un calque d'après l'italien treppiede et s'emploie à côté de la forme empruntée tripiié. Se rapprochent de cette catégorie quelques termes désignant les constructions ou les espaces autour de la maison: portún 'grande porte', štérnę 'citerne', štåle 'étable', córta 'cour', j̇ardín 'jardin'.

Termes désignant des pièces de vêtements, chaussures, literie etc. Le mode de s'habiller a depuis toujours été influencé, en Istrie, par les usages de la Péninsule et 
différentes pièces de vêtement portent, en istroroumain, un nom d'origine italienne: veštid 'vêtement' (forme vénetienne), capelin 'chapeau', baréte 'bonnet' (vén. baréta, Boerio, 64), capót 'manteau', jakéte 'veste, veston' (vén.), bragési 'pantalon' (vén.), bârhån 'jupe', cótule 'robe' (vén.), travérs 'tablier', tinturín 'ceinture, courroie', bârse (sud), bórša (Ž.) 'sac', tacuiinn (sud), tacvín (Ž.) 'portefeuille', šcarpíne (pl.) 'bas de laine', šcafunite (pl.) 'chaussettes' (cf. Skok, 1973, 397) etc. Chaussures: puntål 'semelle', šcapín 'empeigne (de chaussure)' (vén.), spigéte 'lacets'. On peut ajouter aussi les noms de quelques pièces de literie ou de linge qui sont empruntés à l'italien: šugamån 'essuie-main', štramåt 'matelas', lanţún 'drap', cušín 'oreiller'. On peut mentionner ici aussi le mot štraţe 'chiffon' (vén.). Appartiennent au même type d'influence italienne quelques termes pour les parures et les bijoux: cadine 'chaîne, chaînette' (Ž.: cadéniţa, dimin.)(vén.), colår 'collier', urekín (Ž.: rịkín) 'pendant, boucle d'oreille', braţoléto (Ž.) 'bracelet' (vén. brazzalèto, Boerio, 98).

Noms des personnes qui exercent une profession ou une activité. Il y a en istroroumain une liste assez longue de termes de provenance italienne qui désignent une personne exerçant une profession ou une activité quelconque. Même le mot signifiant 'métier', årât, est d'origine italienne. Cela s'explique par le fait que la plupart des métiers et professions modernes, aussi bien que différentes innovations techniques, se sont propagées en Istrie de l'Italie du nord. Nous présentons une liste d'exemples: caligér 'cordonnier' (vén. caleghèr, Boerio, 118), butigér 'boutiquier, marchand' (vén. boteghier, Boerio, 95), camal'ér 'garçon' (it. cameriere), bil'etáío. 'controleur' (it. bigliettaio), bicår 'boucher', cantuniér 'cantonnier', šånto 'tailleur', šårte 'couturière' (à côté des termes croates šiliţe et žnídariţe; it. sarto, sarta), marangún 'menuisier' (it. marangone). Le terme d'origine italienne administrative fåbro (it. fabbro) est employé, dans le parler de Sušnéviţe, comme surnom, tandis que l'emprunt au croate covåt, covåc fonctionne comme appellatif ('forgeron'). Pour la notion 'instituteur, institutrice' les parlers istroroumains du sud ont recours aux emprunts à l'italien maíéštro, maiéštrę, tandis que le parler de Žeinân se sert de l'emprunt à l'allemand Schulmeister (šúmaisster, šúmajisteriţa). Pour la notion 'médecin' l'istroroumain emploie le mot d'origine vénitienne médigo (sud; médiү, Ž.; mèdego, Boerio, 408). Le terme bóšcar 'garde-forestier' est un dérivé croate à partir du substantif emprunté à l'italien boška 'forêt' (istror. bóske, bóške, sud). Les mots stríge, strigón (aussi: strigún) 'sorcière, sorcier' sont des emprunts au vénitien (striga, strigòn, Boerio, 715). L'élément guardijåan, gvardijåan 'gardien' (aussi bien que guårdije 'garde (champêtre), veilleur (de nuit)') est emprunté à l'italien. Le terme qui jouit d'une fréquence relativement élevée dans les textes, manavál '(un) manouevre' (d'habitude sans labialisation de l' /á/ accentué), remonte à l'italien populaire manovale (cf. Zingarelli, $1102,1104)$. Les termes soldåt 'soldat', zandårmi 'gendarmes', secretár 'secrétaire' font penser plutôt aux sources croates dialectales (soldât, žandârmi, sekretâr). Bien que, au point de vue sémantique, ce substantif ne désigne pas une personne active, le mot pâržunér 'prisonnier' (vén. presonier, Boerio, 533) pourrait être classé parmi les mots du présent type à cause de son suffixe. Le terme impiegáto, impiegátę (sud) 
'employé, employée' (avec un [á]) est un italianisme encore vivant mais pas trop fréquent, tandis que l'expression capurål (mağor) (ou capurál, avec un [á]) 'caporal (-chef)' appartient à la langue des hommes qui ont fait leur service militaire à l'époque de l'Italie fasciste.

Noms des exploitations, ateliers, activités professionnelles etc. Les italianismes miniére 'mine (souterraine)' et cåve 'carrière, mine' datent de la fin du XIX ${ }^{\mathrm{e}} \mathrm{s}$., époque où étaient organisées les premières mines modernes en Istrie. Le terme $f a ̊-$ brike 'usine' pourrait être aussi bien un emprunt à l'italien (fabbrica) qu'au croate familier (fàbrika). Quant au mot fabrerijie 'forge', il s'agit d'un dérivé istroroumain à partir du lexème fabbro 'forgeron', mais sur le modèle du mot d'origine croate covaţîle (Ž.: covačíie) 'forge'. L'italianisme gvérę 'guerre' coexiste, dans le sud, avec son synonyme d'origine croate vóiske. Sont d'origine italienne aussi les mots butíge 'boutique, magasin' (cf. it. bottèga) et oštarínie 'bistrot' (cf. it. osteria).

Termes relatifs au domaine administratif, juridique etc. Dans les villages du sud, les sujets possédant des connaissances actives de l'Italien emploient le terme abstrait autoritá 'autorité', qui reste invariable comme en italien et qui n'est pas adapté au point de vue phonétique (on s'attendrait à /au/>/av/, /à/>/å/). Ce terme n'est pas admis dans les parlers croates d'Istrie. Les mots común 'commune' et cunfín 'limite (d'une propriété)', empruntés probablement au vénitien, s'emploient couramment aussi dans les parlers croates. C'est de cunfín que dérive le mot cunfinånti 'voisins' . A côté de l'italianisme campåné 'terrain cultivé, champs' (sud), il faut mentionner le mot paíz 'pays, village' emprunté probablement au frioulan (bien que l'évolution it. paese >paíz ne soit pas à rejeter). Le terme poteštaríle 'palais du podestat' s'applique parfois encore pour désigner le siège de l'administration communale. Le mot pâržún 'prison', employé dans toute l'Istrie et en Dalmatie, est emprunté au vénitien. Bien que le terme généralement admis pour 'argent' soit, dans le sud, le mot pínezi (cr.), on se sert parfois aussi de l'italianisme sóldi, qui fonctionne comme le seul terme dans le parler de Žeiân (šóldi). Le mot préţiie 'prix (convenu)' représente probablement un dérivé istrien sur la base du verbe italien preziare (vén. preziàr). L'it. fitto (vén. fito) a donné en istror. fit 'location, bail'. La forme bandiére 'drapeau' se rattache directement à l'italien bandiera, tandis que la forme bandíra, de Žeiân, s'explique comme pseudo-ikavisme croate. On peut ajouter à ces termes les noms de quelques documents, comme p. ex. matricula (za nevigei) 'matricule (de marin)' et pórte de åmi 'port d'armes' (it. porto d'armi).

Un groupe spécial d'italianismes est représenté par les termes relatifs aux relations sociales, conditions sociales, coutumes etc. Le terme cârstiłåan 'homme' est emprunté au vénitien (cf. cristiàn, crestiàn, Boerio, 209), mais il est courant aussi dans les parlers croates (krstjan, kršćan; Skok, 1972, 197-8) et en frioulan (cf. Pirona, 197). Pour désigner un 'étranger', l'istroroumain se sert - comme la plupart des parlers croates d'Istrie et de Dalmatie - de l'emprunt à l'italien furéšt (it.; vén. forèsto). Pour les notions 'camarade' et 'compagnie', on a recours aux emprunts à l'italien cumpán (rarement cumpån) et cumpanî̉e, à Žeinân cumpåne (it. compagna). Les ter- 
mes uzånţe (sud), užånţa (Ž.) 'coutume', vízita (Ž.) 'visite', bål (sud) 'fête dansante' sont employés aussi dans les parlers croates et remontent à l'it. usanza, visita, ballo (vén. balo).

Termes relatifs à la vie religieuse. Pour exprimer le sens 'messe', l'istroroumain utilise le latinisme croate míse (sud), mísa (Ž.) (cf. Skok, 1972, 430-431), mais les mots miråcul 'miracle', deštín 'destin, destinée' et les termes techniques comme lemózinę 'aumône', funerål 'funérailles', cås 'cercueil, bière (it. cassa da morto)', nónţulo (pl. nónţuli) 'croque-mort, fossoyeur' sont des italianismes (it. miracolo, destino, lemosina, funerale, nonzolo). A ces termes, on pourrait ajouter aussi les mots candéle 'chandelle' et candelír 'chandelier'; ce dernier mot représente un pseudo-ikavisme du même type que bandira (Skok, 1972, 283). La forme ånel 'ange' est une adaptation čakavienne de l'it. àngelo (cf. Skok, 1971, 43; cf. aussi le frioul. àgnul), tandis que vílije 'jeûne, jour maigre' est un emprunt au frioulan (cf. Skok, 1971, $147-8$, s. v. bigla; Pirona, 1277, s. v. vilie). Le terme thimitér 'cimetière' est probablement un latinisme croate, bien que, dans le parler de Sušńéviţę, il pourrait représenter aussi l'aboutissement phonétique de l'it. cimitero (à Sušńéviţę, on ne distingue pas entre les sifflantes et les chuintantes). De la même façon que les Croates d'Istrie, les Istroroumains utilisent assez souvent les formes italiennes des noms de baptême (bérto, románo/rumåno, ğulio, maríla etc.) et des noms de fêtes (Svéti Silvéstro 'la Saint-Sylvestre'). Les formes altérées des expressions italiennes sont parfois à la base des sobriquets de certains Istroroumains (p. ex. banaséra à la base de l'it. $b(u) o n a$ sera).

Termes de parenté et termes relatifs aux relations familiales. Pour dire 'parent, -e, cousin, -e', l'istroroumain du sud utilise l'italianisme cuiín, cuíne (it.: cugino, cugina); le parler de Žeiân a recours au vénitianisme répandu en Istrie zârmån (vén. zerman, Boerio, 811; Skok, 1973, 651). Dans les parlers du sud, l'emprunt au vénitien cuñådo, cuñåde 'beau-frère (mari de la soeur); belle-soeur (femme du frère, soeur du mari)'; dans le parler de Žeiân, cuńådo et cuńåda veulent dire avant toute autre chose 'cousin, -e' ('fils, fille de l'oncle ou de la tante'; signification admise: cuñådo 'beau-frère'; cf. Skok, 1972, 144). Le terme nóno 'grand-père', nóne (Ž.: nóna) 'grand-mère' est un emprunt à l'italien qui a gagné une bonne partie des parlers croates d'Istrie et de Dalmatie. A Žeiân, on emploie à côté de nóno, nóna aussi les expressions čela betânu čåỉe, čå betâra måỉe qui traduisent mot à mot la formule croate čakavienne stari ćaća, stara mama (région de Kastav) qui, à son tour, ne représente qu'un calque approximatif d'après l'allemand Grossvater, Grossmutter. Il sera utile de signaler que c'est à partir du pluriel nóni, et sur le modèle du croate pradjedovi 'ancêtres, aïeux', que certains sujets parlants de l'istroroumain du sud utilisent le calque prénoni ('ancêtres'). Le mot nipót 'petit-fils' représente peut-être un croisement entre le roum. nepót et l'it. nipote (à Žeiân: unúc).

Le terme spózo, spózę (sud) 'jeune marié, fiancé; jeune mariée, fiancée’ (spózi 'nouveaux mariés') est un italianisme de mode et probablement récent. L'istroroumain utilise le mot bárba (parfois aussi bårba) non seulement en tant que terme de 
parenté ('oncle maternel ou paternel') mais bien aussi comme terme de respect pour s'adresser aux hommes adultes. Ce vénitianisme est répandu un peu partout en Istrie et en Dalmatie. Le parler de Žeiân emploie, comme terme de politesse villageoise, le mot cumpår (vén. compàre 'parrain') qui est employé en Istrie et dans certaines partie de Dalmatie (kumpâr); à Žeinân, ce mot a en même temps la signification de 'un tel, (un) type, individu'. Pour exprimer la condition d'un enfant naturel, on se sert de l'italianisme múlo (fém. múliţe) ou baštárdo (fém. baštárdę, baštárda; avec un [á]).

Il faut retenir qu'un certain nombre de termes échappe à toute classification précise, qu'il s'agisse des termes à un sens très général (qui pourraient figurer dans plusieurs catégories) ou bien des éléments qui, à eux seuls, constituent un groupe sémantique à part. Nous signalons quelques substantifs qui n'ont été mentionnés dans aucune des catégories présentées: bånde 'partie', criiåntiųe 'pollitesse', féstę 'fête', finte 'feinte', liberatióne 'libération', parţión 'portion', péze 'poids', púnti 'coutures', pórât 'port', spéze 'frais', štórilie 'conte, histoire', št'óriţe 'fable', våle 'vallée', vóte (sud), vóta (Ž.) 'fois' etc.

Adjectifs. C'est avant tout dans les parlers istroroumains du sud qu'on trouve un nombre relativement élevé d'adjectifs empruntés à l'italien: fin, fíne (Ž.: fína) 'beau, joli' (courant en croate), cunténât, -ntę 'content' (usuel dans les parlers croates d'Istrie), débol, - $e$ 'faible, fragile' (exclusivement dans les parlers istror. du sud; rare dans les parlers croates), dispereit, - $\ell$ 'désespéré', sporc, spórke (Ž.: spórâc, spórca) 'sale', šcúro '(l')obscur' (subst.), líbero, - $\ell$ (sud) 'libre', mercantíle (sud; plutôt individuel), ţénere 'couleur de cendre, gris', drit, - $\varepsilon$ 'droit', țeléste (sud) 'couleur du ciel', tond, $-\varepsilon$

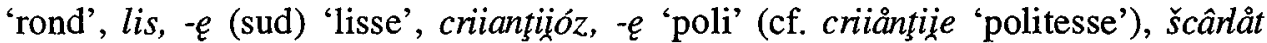
'(l')écarlat' etc. L' adjectif bnínast, - $\varepsilon$ 'brunâtre, brun' représente une forme dérivée à l'aide d'un suffixe croate. Pour exprimer la notion de 'couleur', l'istroroumain utilise un emprunt à l'italien colór (sud), colúr (Ž.).

Verbes. A côté d'un certain nombre de substantifs désignant des actions ou leurs résultats (tir 'tir', caštíg 'châtiement', špuile 'action d'épier, mouchardage' etc.), on trouve en istroroumain aussi plusieurs dizaines de verbes qui remontent à l'italien. Tandis que parmi les substantifs et les adjectifs d'origine italienne on peut trouver des éléments qui ne s'emploient pas en croate, tous les verbes istroroumains dont l'origine italienne est transparente apparaissent normalement aussi dans les parlers croates d'Istrie. Les plus nombreux sont les verbes en éf (cr. en -at, it. en -are), mais il y a aussi quelques verbes en -i (cr. en -it; it. en -ire ou en -ere). A Žeiân, on trouve aussi quelques verbes en - $u \not i j$ (sur le modèle des verbes croates čakaviens en -ovat). Sauf quelques emprunts très anciens (comme p. ex. sparå ( $\breve{Z}$.) 'tirer (un coup de)' < it. sparare), qui proviennent directement de l'italien, tous ces éléments pourraient être traités comme des emprunts à la langue croate. Voici la liste des principaux verbes de ce type: âmbarkéi 'embarquer', coculéi 'dorloter', cumpaniéi 'accompagner', caštigéi 'punir', durẹ́i 'durer', ferméi 'préparer', gambiiélé 'changer', guštéi 'jouir de', makinéi 'moudre', mantréi 'torturer', meritéi 'mériter', moléi 'arrêter', necârgéi 'char-

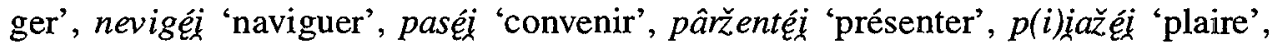


prontéi 'préparer', piturểi 'teindre, badigeonner', peštéi 'fendre; torturer' (aussi spešté́i, popešteì), regulęí 'résoudre, mettre en ordre', rovinéi 'ruiner', ricaméi 'bro-

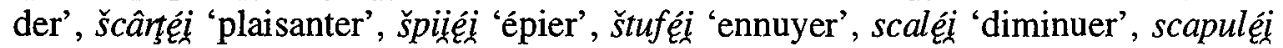
'esquiver, éviter', tamizéi 'tamiser, sasser', timbréí 'marquer', urdinéi 'commander', užét 'avoir l'habitude (de)', t"apéi 'saisir', zecântél '(commencer à) chanter' etc. Les verbes en -í: divertí (se) '(se) divertir', partí 'partir', pošurbi 'siroter', sparí ( $t$ "å) 'disparaître', creši 'grandir'. Les verbes en -úi (Ž.): rešpetúi 'respecter', splodinui 'exploder'. Quoi qu'il en soit, un nombre assez élevé de verbes empruntés suppose un bilinguisme actif, et de longue date, italo-croate d'un côté et croate-istroroumain de l'autre.

La présence de quelques adjectifs numéraux empruntés à l'italien parle en faveur des contacts linguistiques intenses en Istrie. Dans le parler du sud, on trouve le mot mil'år 'mille' qui provient de l'italien migliaro; il faut signaler que l'istroroumain de Žeiân emploie, avec la même signification, le mot croate tisút" ou un emprunt à l'allemand (par l'intermédiaire du slovène) tåvžânt. Le mot cvomår, cvamår '(une) quarantaine, quarante' représente un emprunt à l'istroroman, probablement par l'intermédiaire du croate (cf. Skok, 1972, 251). L'expression dópiỉo déro (type de farine) ne fonctionne en réalité pas comme un numéral.

Les conjonctions ma 'mais' et mácar (Ž.: májar) 'bien que' sont courantes dans les parlers croates d'Istrie. La préposition secóndo 'selon, d'après' semble être un emprunt individuel. Les exclamations d'origine italienne brávo! (et brávi!), ála! conservent la réalisation [á] sous l'accent ce qui prouve qu'elles ne sont pas complètement intégrées dans le système.

Il semble que les adverbes italiens sont entrés en istroroumain en tant qu'unités inanalysables. En même temps, il faut retenir qu'un bon nombre des adverbes istroroumains d'origine italienne est employé aussi dans les parlers croates d'Istrie. Voici une liste des adverbes les plus fréquents et généralement admis: alóra 'alors' (à côté de (a)túnţe, sud), ánke 'aussi, de même', nánke, nánca (< vén. nanca) 'non plus, pas même', alméno 'au moins', béń (ben) 'bien', dónke 'donc', drito '(tout) droit', fórsi 'peut-être', inúšto '(tout) juste, justement', lårgo 'loins' (à Žeinân: depårte, roum.), péna ‘à peine', próprio 'proprement, justement', tánto 'cependant' (it. intanto), $t$ "åro 'très, beaucoup' (< vén. chiaro)

Le nombre des éléments que l'istroroumain a emprunté à la langue italienne (directement ou par l'intermédiaire de la langue croate) est assez élevé et notre liste des italianismes istroroumains pourrait facilement être augmentée. Néanmoins, il faut tenir compte du fait que beaucoup de ces éléments n'apparaissent que rarement dans la langue de tous les jours (surtout à cause de leur caractère plutôt technique) et que leur fréquence dans les textes est réduite. Sextil Puşcariu a démontré qu' un nombre relativement limité des éléments lexicaux hérités du roumain commun représente, dans les textes de Sušńéviţe, $82 \%$ des occurences lexicales, tandis que les nombreuses unités lexicales empruntées au croate, à l'italien etc. ne correspondent 
qu'à $18 \%$ des occurences dans les textes (Puşcariu, 1926, 220-221). D'après nos analyses statistiques, le rapport entre les éléments lexicaux hérités et les emprunts serait comme il suit: a) Textes de Sušnéviţę: éléments hérités du roumain commun $77,4 \%$ des occurences dans le texte, éléments slaves (plus particulièrement croates) $-15,7 \%$, éléments italiens $-6,9 \%$; b) Textes de Žeiân: éléments hérités du roumain commun $-81,6 \%$, éléments slaves (plus particulièrement croates) $-16,4 \%$, autres éléments (y compris l'élément italien) - 2,0 \% (Kovačec, 1984, 581).

Puşcariu affirme que l'élément lexical italien en istroroumain est représenté avant tout par des emprunts au vénitien (Puşcariu, 1926, 223). Une étude minuteuse du problème pourrait, peut-être, prouver que les emprunts au dialecte vénitien constituent la majeure partie des italianismes anciens. Il faut cependant mettre en valeur le fait que le dialecte vénitien et l'italien standard du type toscan, en tant que deux variétés de la même langue, ont en commun une quantité considérable du matériel linguistique et il n'est pas toujours possible de faire une distinction systématique entre leurs éléments. Dans les cas où il est possible de faire une distinction formelle claire entre les formes lexicales vénitiennes et celles qui proviennent de l'italien standard du type toscan (cf. Tekavčić, I, 1972, 180-191, 193, 275, 281, 285 et passim), on constate que le nombre des éléments du lexique istroroumain qui remontent à l'italien standard dépasse de beaucoup le nombre des éléments du type vénitien. Les éléments de provenance frioulane et istroromane ne constituent que des cas isolés. Quoi qu'il en soit, il n'y a pas de doute que l'istroroumain a emprunté au vénitien les termes suivants: catóme, brúla, bízi, fažó, petâršín, mérlin, capúz, túkę, țukéte, cucúmari, intråde, fịorét, túcor, manéstre, maréndę, stúmig, batúde, núzine, duzíne, šéšula, ferål, medizúle, bóțe, fugére, pošådę, cåmare/cåmbra, fogulér, veštíd, baréte, cadinę/cadénița, caligér, butigér, médigo/médiү, strige, pâržunér, pâržún, cârstiīån, zârmån, cuńådo, cuñådę, nóno, nóne, cumpår, nánca. Il est cependant difficile de faire une distinction entre les éléments qui représentent des emprunts directs au vénitien et ceux qui sont entrés en istroroumain par l'intermédiaire du croate.

Isolé dans la montagne, le village de Žeinân avait peu de contacts avec le reste du pays. Les rapports que ses habitants entretenaient avec les populations de langue italienne n'étaient que sporadiques et superficiels. Dans les villages istroroumains du sud, au contraire, les contacts avec les groupes italophones ont depuis toujours été plus réguliers et plus intenses. Depuis le milieu du XIX ${ }^{\mathrm{e}}$ s., les hommes des villages istroroumains du sud, très souvent, $\mathrm{s}$ 'embarquaient à bord des bateaux de commerce où l'italien était utilisé normalement comme langue de métier et langue 'interethnique'. C'est pourquoi dans les villages du sud il y a toujours eu un certain nombre de personnes capables de se servir activement de la langue italienne. Etant donné les circostances que nous venons d'esquisser, l'istroroumain du sud a emprunté à l'italien un nombre de mots presque trois fois plus élevé de ce qu'a fait l'istroroumain du nord.

Une partie au moins des éléments d'origine italienne dans le vocabulaire istroroumain accuse un caractère éphémère et assez instable, et il est parfois difficille de 
porter un jugement sur la question s'il s'agit d'un italianisme généralement accepté ou bien d'un emprunt individuel ou occasionnel. Il faut tenir compte aussi du fait que le nombre et la nature des emprunts à l'italien dépendent du caractère du texte où ils apparaissent ou bien du sujet dont on parle. Néanmoins, il arrive souvent que l'istroroumain du sud se serve d'un emprunt à l'italien là où l'istroroumain de Žeinân utilise un élément d'une autre provenance. Nous avons analysé ailleurs les différences lexicales entre les deux variétés de l'istroroumain (Kovačec, 1981, 57-83; 1984, 584-6), et nous allons présenter ici quelques différences qui reposent sur la présence d'un emprunt à l'italien dans les parlers du sud et son absence dans le parler de Žeiân.

Pour quelques notions de temps, les parlers du sud utilisent des emprunts à l'italien, comme p. ex. otóbre (à côté du croate mihósńacu) 'octobre', setimåne 'semaine', primavére (à côté des mots croates prolitt"e, mládo léto) 'printemps', autúno (à côté du cr. lésen) 'automne', tandis que le parler de Žeiân se sert des mots hérités du roumain commun (tómna 'automne', primavéra 'printemps') ou bien des emprunts au croate ( $t$ "édân 'semaine', otóbâr 'octobre', qui est un emprunt à l'italien adapté au point de vue phonétique et morphologique). On retrouve le même rapport entre les deux variétés de l'istroroumain pour toute une série de notions: stéurę/stévrę 'impôt', libri 'livres', clåse 'classe', maiéstro (-e) 'instituteur (-trice)', månig, (le) 'manche', sapún 'houe, hoyau', butíro, búro (à côté du cr. måslo) 'beurre', mil'år 'mille, millier' etc. dans le parler de Sušńevițe, alors que le parler de Žeiân utilise des emprunts au croate ou bien des éléments hérités du roumain commun: pórez 'impôt' (cr.), crírye 'livres' (cr.), råzred 'classe' (cr.), šúmaister (-itga) 'instituteur (-trice)' (cr./sl. < all.), dâržâc '(le) manche' (cr., bien que måniү ne soit pas inconnu), motíca 'houe, hoyau' (cr.), unt 'beurre' (roum.), tisút" (cr.) ou tåvžânt 'mille' (all., par l'intérmédiaire du slovène) etc. De même que dans le cas de måni , les parlers du sud utilisent le mot italien campåne 'champs (campagne)' là où le parler de Žeiân se sert du mot croate pól'e, mais le terme campåné n'est pas tout à fait inconnu au nord. On trouve aussi les cas où, pour la même notion, le sud et le nord emploient un élément d'origine italienne, mais qui était introduit en istroroumain par voies différentes: cåmare 'chambre' (sud), cåmbra (Žeiân).

Dans les deux variétés de l'istroroumain, se constituent parfois des paires de synonymes sur la base d'un emprunt au croate (commun aux deux variétés) et un emprunt à l'italien dans le sud, à l'allemand à Žeiân. On trouve p. ex. dans le parler de Sušnéviţe les synonymes dår (cr.) et pârzént (it.) et à Žeiân dår et šenc 'cadeau' (all.; le mot šenk est courant dans les parlers croates de la Cićarija et dans les parlers slovènes limitrophes). Dans d'autres cas, au lieu d'un emprunt à l'italien dans le sud (surtout pour les adjectifs, les adverbes et les verbes) le parler de Žeiân utilise un mot emprunté au croate, à l' allemand etc. Par exemple, aux éléments d'origine italienne cunténât 'content, satisfait', débol 'faible, fragile', țeléste/čeléste 'bleu clair, couleur du ciel' etc., péna 'à peine', alméno 'au moins', fórsi 'peut-être' etc., caractéristiques pour les parlers du sud, l'istroroumain de Žeiân utilise de préférence les éléments d'une origine différente: zadovól'ân 'content', švóhân 'faible' (all.), víret 'bleu' 
(roum.) etc., cómat" 'à peine', bárem 'au moins', mórke 'peut-être etc. On peut ajouter à cette liste plusieurs verbes, comme p. ex. spari t"å 'disparaître', duréi 'durer', capí 'comprendre', scometéi (it. scommettere), 'parier', parit"éi (vén. parechiàr), prontéi 'préparer' etc. dans les villages du sud, auxquels correspondent dans le parler de Žeiân les verbes nestani 'disparaître', traięí 'durer', anţelęže (roum.), razumi (cr.) 'comprendre' (dans le sud, aussi le mot rezumí), vadl'éi (se) 'parier', priproví/pripravl'úi 'préparer' etc. Dans le parler de Žejân, les emprunts à l'italien s'adaptent normalement aux normes morphologiques de l'istroroumain (p. ex. médi ' 'médecin', pl. médiž), et les cas où cette adaptation n'est que partielle sont plutôt rares (p. ex. nóno 'grand-père'). Dans les parlers du sud, au contraire, un nombre important d'emprunts semble avoir influencé la structure morphologique de l'istroroumain (médigo, nóno, autúno, šårto; líbero, - $e$ etc.; v. infra).

Sont tout à fait exceptionnels les cas où le parler de Žeiân se sert d'un emprunt à l'italien (p. ex. šóldi 'argent', par l'intermédiaire du croate), et que l'istroroumain du sud utilise un élément d'origine croate (pinez 'argent'; le mot sóldi est admis aussi dans le sud). A un examen plus attentif de la forme des emprunts à l'italien, on peut se rendre compte que les unités de ce type se comportent de différentes manières. Il y a des emprunts qui se soumettent systématiquement aux normes phonétiques et morphologiques de l'istroroumain. Par exemple, dans les villages du sud, quelques individus qui ont terminé une école italienne, ou qui entretiennent des contacts réguliers avec les italophones, se servent parfois, en parlant istroroumain, des mots čitá 'ville' (au lieu de gråd, cr.), autoritá 'autorité' (cr. vlast). On peut constater que dans des cas semblables $/ \check{c} /$ de čitá ne se réalise jamais comme $[t]$ ([ts]), un phénomène auquel il faudrait s'attendre dans un parler, comme celui de Sušnéviţę, qui ne connaît pas d'opposition entre les sifflantes et les chuintantes (Kovačec, 1971, 72). D'une manière semblable, les deux mots ont systématiquement la réalisation [á] (čitá, autoritá, de même que impiegáto, -e, ánke, bárem, mácar, brávo, nánca, tánto etc.) et le mot autoritá n'admet pas le passage /aun/ > /av/ (il n'y a pas de réalisations *titå, *avtoritå). Ces deux mots ne changent pas au pluriel. Les éléments čitá et autoritá, qui n'appartiennent pas à la langue générale, fonctionnent selon des règles particulières.

Bien que la majeure partie des emprunts istroroumains à l'italien se soient adlaptés à la morphologie istroroumaine, il y a des emprunts qui ont apporté des modifications au système morphologique istroroumain. Dans le parler de Žeiân, le substantif avec le sens 'médecin' est complètement intégré dans le système morpho-

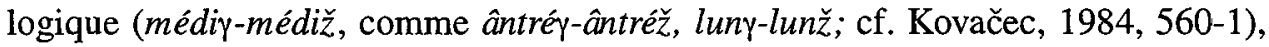
alors qu'à Sušnéviţę on trouve les formes médigo (rarement médig) pour le singulier et médiz/ médiž pour le pluriel. D'une manière semblable, le mot emprunté au vénitien, cuńådo, a au pluriel cuñåz (comme suséd-suséz; ibid.). Malgré le fait que les substantifs dont il est question ici se sont intégrés dans les schèmes morphologiques existants, il est nécessaire de relever plusieurs faits importants. Premièrement, c'est grâce aux emprunts à l'italien que l'istroroumain a introduit une nouvelle variante du monème 'masculin singulier' /-o/ (aux variantes déjà existantes $/-\phi,-e,-u /$ : lup- $\phi$, 
frait-e, socr-u on a ajouté la variante /-o/: cuńåd-o, non-o, nónţul-o, impiegåt-o, gob-o, mul-o, spoz-o etc.). Deuxièmement, c'est grâce à l'introduction des pluriels italiens en /-i/ (non-i, nóntul-i, impiegåt-i, gob-i, mul-i, spoz-i etc.) que le monème/-i//'masc. pl.' - qui, jusque là, n'était possible qu' après un groupe consonantique (socr-i, hlåptt-i) cesse d'être conditionné par le contexte phonétique. Enfin, la troisième conséquence importante est que le système des alternances consonantiques, qui servent pour l'expression du nombre, ne s'applique pas systématiquement à ce type de substantifs et cesse, par conséquent, de fonctionner comme un automatisme: nónţuli, impiegáti,

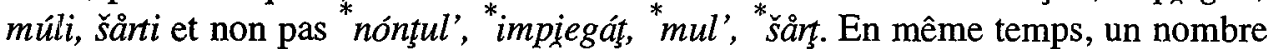
plus élevé des cas où le signifiant pour 'masc. pl.' est /-i/ contribue à l'effacement de la distinction 'indeterminé' - 'déterminé' (lup lúpi: nóni nóni) à la fin des substantifs. Selon le même modèle, les adjectifs empruntés à l'italien (comme líbero, $-\varepsilon$ ) accusent les mêmes caractéristiques morphologiques (líber- $o,-\ell,-i,-e$ en face du type bur $\emptyset$, $,-e,-\varnothing,-e)$. Quoi qu'il en soit, les emprunts à l'italien ont modifié les modèles de l'expression du genre et du nombre des substantifs et des adjectifs. Il est nécessaire pourtant de souligner que les substantifs de ce type sont peu nombreux dans le parler de Žeiân et que les adjectifs du type líbero, $-\varepsilon$ n'y existent pas.

On sait bien que la variété septentrionale de l'istroroumain conserve assez bien la déclinaison synthétique des substantifs et des adjectifs, alors que dans les parlers du sud les fonctions du substantif dans la phrase s'expriment presque exclusivement à l'aide des prépositions ou autres éléments préposés (Kovačec, 1984, 567-9). Bien qu'il soit difficile d'affirmer que cette l'évolution, dans le sud, est la conséquence d'une influence italienne, et non pas le résultat d'une évolution interne, l'influence italienne n'est pas à rejeter.

Enfin, une importante innovation dans le système verbal des parlers istroroumains du sud ne peut être expliquée que par l'influence de la langue italienne. Le verbe istroroumain veri 'venir' a développé, sous l'influence italienne, deux significations nouvelles caractéristiques pour les dialectes italiens du nord. D'un côté, le verbe istroroumain veri a développé le sens 'devenir' (it. venire = 'diventare'): verí bogåt 'devenir riche' ('s'enrichir'), verí betâr 'devenir vieux ('vieillir'). Ce calque est courant aussi dans le parler de Žeiân. De l'autre côté, si l'italien exprime normalement le passif à l'aide de l'auxiliaire essere et du participe (Tekavčić, 1972 (II), 318-320), c'est sur le modèle de l'italien du nord, où le passif est exprimé aussi à l'aide du verbe venire en tant que verbe auxiliare (Rohlfs, 1970, 129; Zingarelli, 2125), que l'istroroumain du sud a développé un moyen nouveau pour exprimer le passif à l'aide de l'auxiliaire verí: våca virít-a uţíse 'la vache a été tuée', a côté du type våca fóst-a uţise (it. la vacca è stata uccisa). Il est cependant indispensable de mettre en évidence au moins deux restrictions à cette possibilité. En italien, ce type de passif est restreint aux 'temps simples' (Zingarelli, 2125), alors qu'en istroroumain la construction dont il est question ici peut être employée dans n'importe quel temps. Dans les parlers istroroumains du sud où elle apparaît, cette tournure n'est pas généralement employée et elle n'est même pas admise par tous les sujets parlants: 
nous l'avons enregistrée dans la parole de quelques personnes ayant des connaissances actives de l'italien.

Un contact linguistique intense et durable, et qui se réalisait dans des conditions favorables, a eu pour résultat un grand nombre d'emprunts lexicaux en istroroumain. Cette masse d'emprunts a provoqué d'importantes restructurations dans le système grammatical de la langue emprunteuse. Au cours de notre analyse, il a été relativement facile d'établir les correspondances entre les éléments istroroumains et les éléments italiens dont ils sont issus. Mais l'étude des voies par lesquelles les mots italiens ont pénétré en istroroumain se heurte à de nombreuses difficultés, surtout à cause du fait que beaucoup de chaînons indispensables pour retracer l'histoire des mots font défaut. Ce n'est qu'un atlas linguistique du domaine istrien, comme celui proposé par le professeur Tekavčić, qui pourrait suppléer ce manque.

Notes

Par opposition au dialecte vénitien, nous désignons par le terme italien (it.) aussi bien l'italien standard du type toscan ('italien littéraire et officiel') que la langue italienne en général.

Valeur des signes particuliers dans notre notation phonétique. Voyelles: e voyelle palatale la plus ouverte (æ, ä); å - voyelle vélaire la plus ouverte, labialisée. Semi-voyelles: $\mathbf{i}$ - semi-voyelle palatale (et fricative palatale sonore); $\mathbf{u}-$ semivoyelle labio-vélaire. Consonnes: sifflantes: $\mathrm{t}(=\mathbf{t s}), \mathbf{d}(=\mathbf{d z}), \mathbf{s}, \mathbf{z} ;$ chuintantes: $\check{\mathbf{c}}(=\mathbf{t} \breve{s})$,

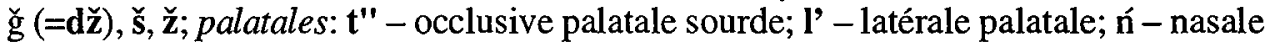
palatale.

\section{BIBLIOGRAPHIE}

Boerio (1856): Giuseppe Boerio, Dizionario del dialetto veneziano, Seconda edizione aumentata e corretta, Venezia;

Flora, 1975: Radu Flora, Gli italianismi nell'istroromeno, Italica Belgradensia, I, Belgrado (pp. 45-59, 4 cartes);

Kovačec, 1968: August Kovačec, Observations sur les influences croates dans la grammaire istroroumaine, La Linguistique, 1968/I, Paris (pp. 79-115);

Kovačec, 1971: A. Kovačec, Descrierea istroromânei actuale, Bucureşti (230 p.);

Kovačec, 1981: Les différences lexicales entre l'istroroumain du nord et l'istroroumain $d u$ sud, Studia Romanica et Anglica Zagrabiensia (SRAZ), XXVI (1-2), Zagreb (pp. 57-83);

Kovačec, 1984: A. Kovačec, Istroromâna, Tratat de dialectologie românească, Craiova (pp. 550-591; avec une carte, p. 970); 
Petrovici et Neiescou, 1965: Emil Petrovici et Petru Neiescou, Persistance des îlots linguistiques, Revue roumaine de linguistique, tome X, $\mathrm{N}^{\circ} 4$, Bucarest (pp. 351374);

Pirona (1967): Giulio Andrea Pirona, Ercole Carletti, Giov. Batt. Corgnali, Il nuovo Pirona, Vocabolario friulano, $2^{\mathrm{a}}$ edizione, Udine;

Puşcariu, 1926: Sextil Puşcariu, Studii istroromâne, În colaborare cu M. Bartoli, A. Belulovici şi A. Byhan, II, Întroducere-Gramatică-Caracterizarea dialectului istroromân, Bucureşti;

Puşcariu, 1929: S. Puşcariu, Studii istroromâne, III, Bibliografie critică-Listele lui Bartoli-Texte inedite-Note-Glosare, Bucureşti:

Rohlfs, 1970: Gerhard Rohlfs, Grammatica storica della lingua italiana e dei suoi dialetti, Sintassi e formazione delle parole, Traduzione di T. Franceschi e M. Caciagli Fancelli, Torino;

Skok, 1971: Petar Skok, Etimologijski rječnik hrvatskoga ili srpskogajezika, Uredili akademici Mirko Deanović i Ljudevit Jonke, Suradjivao u predradnjama i priredio za tisak Valentin Putanec, JAZU, Zagreb, Knjiga prva, A-J;

Skok, 1972: P. Skok, id., Knjiga druga, K-poni ${ }^{1}$;

Skok, 1973: P. Skok, id., Knjiga treća, poni ${ }^{2}-\check{Z}$;

Tekavčić, 1972: Pavao Tekavčić, Grammatica storica dell'italiano, Volume I: Fonematica; Volume II: Morfosintassi; Volume III: Lessico, Bologna;

Tekavčić, 1976: P. Tekavčić, Per un atlante linguistico istriano (Con speciale riguardo ai dialetti istroromanzi), Studia Romanica et Anglica Zagrabiensia (SRAZ), 41-42, Zagreb (pp. 227-240);

Zingarelli (1989): Nicola Zingarelli, Vocabolario della lingua italiana - Il nuovo Zingarelli, Undicesima edizione, a cura di Miro Dogliotti e Luigi Rosiello, Bologna.

\author{
Povzetek \\ ITALIJANS KE LEKSIKALNE PRVINE V ISTROROMUNŠČINI
}

\begin{abstract}
Po kratki predstavitvi kulturnozgodovinskih in sociolingvističnih dejstev, ki se tičejo usode istroromunščine, so navedeni italianizmi $v$ istroromunščini, in sicer neposredne in posredne izposojenke iz različnih zvrsti italijanskega jezika. Samostalniki so razvrščeni po pomenskih poljih, druge izposojenke po besednih vrstah. Posebej se tehta vloga beneškega narečja in pa odnos hrvaškega in italijanskega jezika (hrvaščina je lahko tudi posrednik). V luči izposojanja iz italijanskega besedišča so predstavljene tudi razlike med severno in južno varianto istroromunščine.

Članek se končuje $\mathrm{z}$ analizo posledic, ki jo je izposojanje leksikalnih prvin imelo v nekaterih delih istroromunskega slovničnega sestava.
\end{abstract}

